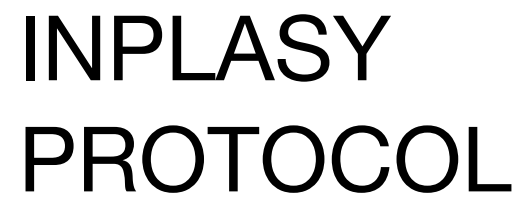

To cite: Yan et al. A comparison of the efficacy and safety of traditional Chinese medicine external treatment for the hyperemesis gravidarum: $A$ protocol for systematic review and network metaanalysis. Inplasy protocol 202090089. doi:

10.37766/

inplasy2020.9.0089

Received: 25 September 2020

Published: 25 September 2020

Corresponding author: Ru-gen Yan

13793196710@163.com

Author Affiliation:

Shandong University of Traditional Chinese Medicine

Support: No.

Review Stage at time of this submission: Data analysis.

Conflicts of interest: No.

\section{A comparison of the efficacy and safety of traditional Chinese medicine external treatment for the hyperemesis gravidarum: A protocol for systematic review and network meta-analysis}

Yan, RG1'; Zhan, JY2; Liu, GX33; Li, CZ; Cai, PP5; Chen, Y6; Cao, HZ7.

Review question / Objective: Type of studies. Regardless of whether blinding is used or not, all RCTs of TCM external treatment of HG will be included. Clinical trials without a control group will be excluded. Type of participants. Pregnant women who were clinically diagnosed with HG, regardless of age or race will be included. The diagnosis of HG should meet the diagnostic criteria of the guidelines for HG established by the ACOG. Interventions and comparators. We will include all type of TCM external treatment for HG such as acupuncture, acupoint pressing, acupoint sticking, acupoint injection. Patients in both groups were treated with intervention based on routine rehydration therapy, duration of treatment unlimited. Outcomes indicators. Primary outcomes. The primary outcomes of what we desperately want is the intensity and severity of nausea and vomiting. Secondary outcomes.Quality of life, Adverse outcomes of pregnant women, Adverse outcomes of fetal, Duration of hospitalization. Method:In this study, English and Chinese literatures that meet the requirements will be searched in EMBASE, PubMed, Cochrane Library, Web of Science, CnKI, VIP, Wanfang, and CBM. Regardless of whether blinding is used or not, all randomized controlled trials(RCTs) using TCM external treatment for the HG will be included. Next, STATA 16.0 will be used to conduct a series of pairwise meta-analysis.WinBUGS 1.4.3 and Stata 16.0 will be used to conduct Bayesian network meta-analysis to evaluate the relative results of different TCM external treatment. The quality included in the study will be evaluated through the classification of Grading of Recommendations Assessment, Development and Evaluation(GRADE).

INPLASY registration number: This protocol was registered with the International Platform of Registered Systematic Review and MetaAnalysis Protocols (INPLASY) on 25 September 2020 and was last updated on 25 September 2020 (registration number INPLASY202090089).

\section{INTRODUCTION}

Review question / Objective: Type of studies. Regardless of whether blinding is used or not, all RCTs of TCM external treatment of HG will be included. Clinical trials without a control group will be excluded. Type of participants. Pregnant women who were clinically diagnosed with HG, regardless of age or race will be 
included. The diagnosis of HG should meet the diagnostic criteria of the guidelines for HG established by the ACOG. Interventions and comparators. We will include all type of TCM external treatment for HG such as acupuncture, acupoint pressing, acupoint sticking, acupoint injection. Patients in both groups were treated with intervention based on routine rehydration therapy, duration of treatment unlimited. Outcomes indicators. Primary outcomes. The primary outcomes of what we desperately want is the intensity and severity of nausea and vomiting. Secondary outcomes.Quality of life, Adverse outcomes of pregnant women, Adverse outcomes of fetal, Duration of hospitalization. Method:In this study, English and Chinese literatures that meet the requirements will be searched in EMBASE, PubMed, Cochrane Library, Web of Science, CnKI, VIP, Wanfang, and CBM. Regardless of whether blinding is used or not, all randomized controlled trials(RCTs) using TCM external treatment for the HG will be included. Next, STATA 16.0 will be used to conduct a series of pairwise metaanalysis.WinBUGS 1.4 .3 and Stata 16.0 will be used to conduct Bayesian network meta-analysis to evaluate the relative results of different TCM external treatment. The quality included in the study will be evaluated through the classification of Grading of Recommendations Assessment, Development and Evaluation(GRADE).

Condition being studied: The frequent nausea and vomiting, inability to eat, and fluid imbalance caused by hyperemesis gravidarum (HG) seriously impact the quality of life of pregnant women. In some serious cases, it is even necessary to terminate the pregnancy, which threatens the life of the pregnant woman. A great many of studies have proved that HG can be effectively treated by traditional Chinese medicine(TCM) external treatment. Nevertheless, its relative effectiveness and safety haven't yet been confirmed because of the variety of TCM external treatment. Therefore, we will use the method of network meta-analysis to verify the effectiveness and safety of different types of TCM external treatment for the HG.

\section{METHODS}

Participant or population: Pregnant women who were clinically diagnosed with HG, regardless of age or race will be included. The diagnosis of HG should meet the diagnostic criteria of the guidelines for HG established by the ACOG. Patients are not invited to participate in the writing or editing of this article for readability or accuracy.

Intervention: The experimental group will include all type of TCM external treatment for HG such as acupuncture, acupoint pressing, acupoint sticking, acupoint injection.

Comparator: The control group was treated with antiemetic drugs or different types of TCM external treatments. Patients in both groups were treated with intervention based on routine rehydration therapy, duration of treatment unlimited.

Study designs to be included: The agreement was designed in accordance with the "the Preferred Reporting Items for Systematic Review and Meta-analysis Protocols" [18] and registered with inplasy. The results of the systematic review and network meta-analysis are expected to be published in recognized journals. Since this is a systematic literature study, there is no need to apply for ethical review.

Eligibility criteria: Type of studies. Regardless of whether blinding is used or not, all RCTs of TCM external treatment of HG will be included. Clinical trials without a control group will be excluded. The language is restricted to English and Chinese. Type of participants. Pregnant women who were clinically diagnosed with $H G$, regardless of age or race will be included. The diagnosis of HG should meet the diagnostic criteria of the guidelines for HG established by the ACOG. Patients are not invited to participate in the writing or editing of this article for readability or accuracy.Interventions and comparators. The experimental group will include all type of TCM external treatment for HG such as acupuncture, acupoint pressing, acupoint 
sticking, acupoint injection. The control group was treated with antiemetic drugs or different types of TCM external treatments. Patients in both groups were treated with intervention based on routine rehydration therapy, duration of treatment unlimited.

Information sources: We will conduct a comprehensive search in the PubMed, EMBASE, Web of Science and Cochrane libraries to determine the available English data. In addition, we will also search for Chinese e-bibliographic database resources, mainly including China national knowledge infrastructure(CNKI), Weipu database(VIP), Wanfang database, and China Biology Medicine(CBM). Furthermore, we will also search for ongoing or unpublished trials such as the International Clinical Trials Registry Platform, the NIH Clinical Trails, and the Chinese Clinical Register.

Main outcome(s): Primary outcomes. The primary outcomes of what we desperately want is the intensity and severity of nausea and vomiting(Frequency and duration of nausea and vomiting). The severity of nausea and vomiting was evaluated by the Modified Pregnancy-Unique Quantification of Emesis and Nausea (PUQE), which is a reliable and effective method. The score of the PUQE index is closely related to the quality of life.

Additional outcome(s): (1)Quality of life: The physical symptoms, aggravating factors, fatigue, emotion and limitation were scored by the pregnancy Nausea and Vomiting of Pregnancy Quality of Life questionnaire (NVPQOL). The lower the score, the higher the quality of life. (2)Adverse outcomes of pregnant women: weight loss, gestational hypertension and preeclampsia, etc. (3)Adverse outcomes of fetal: spontaneous abortion, stillbirth, premature infants, low birth weight infants, etc. (4)Duration of hospitalization.

Quality assessment / Risk of bias analysis: The quality included in the study will be evaluated through the classification of Grading of Recommendations Assessment, Development and Evaluation(GRADE).
There will be 2 reviewers evaluate the quality of the included studies independently using Cochrane Handbook (version 5.2.0).

Strategy of data synthesis: Stata 16.0 software will be used for analysis, and the measurement data are evaluated by mean difference (MD), the odds ratio (OR) will be used to evaluate enumeration data. Each effect size will be given its estimated value and $95 \%$ confidence interval. The $\mathrm{X} 2$ test in Stata 16.0 will be used to evaluate the $p$ value between the results of each study, and 12 will be used to quantitatively determine the size of heterogeneity. If 120.05 , it means that there is no heterogeneity between the studies, and the fixed effects model can be used; If $12>50 \%$, $p<0.05$, it means that there is heterogeneity between the studies, then the source of heterogeneity should be analyzed, such as age, disease course, etc.

Subgroup analysis: If the studies included are highly heterogeneous, we will conduct a subgroup analysis to explore age, race, different types of TCM external treatment, treatment time, methodological quality, etc.

Sensibility analysis: For the purpose of ensuring the credibility of the research results, we will analyze their sensitivity by the following methods : (1).Change of the inclusion criteria. (2). Exclusion of lowquality or open studies. (3).Analysis of the same data using different statistical methods/models.

\section{Country(ies) involved: China and USA.}

Keywords: traditional Chinese medicine external treatment; hyperemesis gravidarum; network meta-analysis; protocol; systematic review.

\section{Contributions of each author:}

Author 1 - Ru-gen Yan - Conceptualization, Investigation, Methodology, Resources, Writing-original draft, Writing-review \& editing.

Author 2 - Jun-yi Zhan - Data curation, Investigation, Resources, Writing-original draft. 
Author 3 - Gong-xue Liu - Data curation, Resources, Software operation.

Author 4 - Chang-zhong Li Conceptualization, Methodology, Supervision.

Author 5 - Ping-ping Cai Conceptualization, Methodology, Supervision.

Author 6 - Yin Chen - Data curation, Resources.

Author 7 - Huan-ze Cao - Software operation. 